\title{
Macrostate and Microstate of EEG Spatio-Temporal Nonlinear Dynamics in Zen Meditation
}

\author{
Pei-Chen Lo', Wu Jue Miao Tian², Fang-Ling Liu' \\ ${ }^{1}$ Institute of Electrical and Control Engineering, National Chiao Tung University, Taiwan \\ ${ }^{2}$ Shakyamuni Buddhist Foundation, Taiwan \\ Email:pclo756@g2.nctu.edu.tw
}

How to cite this paper: Lo, P.-C., Tian, W.J.M. and Liu, F.-L. (2017) Macrostate and Microstate of EEG Spatio-Temporal Nonlinear Dynamics in Zen Meditation. Journal of Behavioral and Brain Science, 7, 705-721.

https://doi.org/10.4236/jbbs.2017.713046

Received: November 7, 2017

Accepted: December 11, 2017

Published: December 14, 2017

Copyright $\odot 2017$ by authors and Scientific Research Publishing Inc. This work is licensed under the Creative Commons Attribution International License (CC BY 4.0).

http://creativecommons.org/licenses/by/4.0/

\begin{abstract}
Macrostate and microstate characteristics of interregional nonlinear interdependence of brain dynamics are investigated for Zen-meditation and normal resting EEG. Evaluation of nonlinear interdependence based on nonlinear dynamic theory and phase space reconstruction is employed in the 30-channel electroencephalographic (EEG) signals to characterize the functioning interactions among different local neuronal networks. This paper presents a new scheme for exploring the microstate and macrostate of interregional brain neural network interactivity. Nonlinear interdependence quantified by similarity index is applied to the phase trajectory reconstructed from multi-channel EEG. The microstate similarity-index matrix (miSIM) is evaluated every 5 millisecond. The miSIMs are classified by $K$-means clustering. The cluster center corresponds to the macrostate SIM (maSIM) evaluated by conventional scheme. Zen-meditation EEG exhibits rather stationary and stronger interconnectivity among frontal midline regional neural oscillators, whereas resting EEG appears to drift away more often from the midline and extend to the inferior brain regions.
\end{abstract}

\section{Keywords}

Electroencephalograph (EEG), Nonlinear Interconnectivity, Microstate, Macrostate, Zen Meditation

\section{Introduction}

Method based on the chaotic nonlinear dynamical modeling is developed to explore the microstate and macrostate phenomena of brain functioning. Instead of 
characterizing the portrait of brain potential mapping at a time instant [1] [2], a microstate of the system could be viewed as an instantaneous snapshot of the nonlinear dynamical activities of all brain neurons in the millisecond scale. The microstate reveals the momentary glint of brain atlas in different physiological, psychological, or consciousness states [3]. The macrostate is characterized by the probability distribution of the statistical ensemble of all possible microstates. A macrostate can be viewed as the neuronal-dynamic equilibrium state of the macroscopic properties of the interregional (active/passive) activity distributed over a given time span. In brain dynamics, local neural networks collaborate together to accomplish specific tasks [4]. Each local neural-network module simultaneously plays the active (source) and passive (sink) role while sending and receiving electrical signals during the task. EEG measures the spontaneously aggregated electrical potentials generated by various types of neural oscillations of the brain down to millisecond-range temporal resolution. The brain dynamic macrostate becomes more spread out with the increase of various microstates during a time interval [5]. A microstate occurring with higher possibility indicates a better chance for the system to settle in such a specific microstate.

Scientific exploration has corroborated the effectiveness of meditation on health and well-being. Particular evidence includes the improvement of cardiovascular functions, immunity, and hormone regulation. Moreover, meditation makes positive changes in the brain, including the positive emotional states, better stress manipulation, enhanced mindful attention, noticeable reduction in anxiety and depression [6] [7] [8] [9]. Meditation is a unique state of transcendental consciousness beyond the ordinary mental process and consciousness. Most meditation practices are aimed to better manipulate the mind, brain function and physical state through mindfulness concentration and respiratory regulation. Having been adopted as the complementary medical practices, meditation inspired more scientific studies on its physiological and mental effects [10] [11] [12]. Meditation researches are mostly focused on Yoga and transcendental meditation from India, Japanese Zen, and Tibetan Buddhism [13] [14]. However, there is a lack of studies with respect to the orthodox heart-to-heart imprint sealing (HHIS) Zen meditation, of which the practitioners aim to liberate the true wisdom, Bodhi, inside the heart. HHIS Zen meditation was promulgated to mainland China by the $28^{\text {th }}$ Zen patriarch Bodhidharma around AC 520 and transmitted to the current Zen patriarch Wu Jue Miao Tian. Patriarch Wu Jue Miao Tian for the first time disclosed the ten-mailuns system capable of effectively purifying the negative memories in the brain and converting the clinging brain into tranquil, detached brain [15]. It has been proposed that activation of ten mailuns locating at the glands or nerve plexuses can facilitate Zen meditation. HHIS Zen meditation, completely distinct from the other meditation techniques, follows the heart-dominating principle while meditations are mostly mind-cultivating practices. It is supposed that through years of Zen meditation, practitioners have their brain functions totally reformed into a so-called detached brain dominated by Bodhi. In this study, we investigate the Zen-medi- 
tation EEG to objectively study the putative brain reformation process. EEG applications have become favorable since 1970s because of its advantages of good spatiotemporal resolution, economy, safety and convenience. Most of all, more scientific evidences of EEG variations have been obtained in different physiological, pathological, conscious and mental states in accordance with various temporal, spatial and spectral EEG characteristics [16] [17] [18] [19].

To explore the spatial interactions among local neural networks under Zen meditation, methods developed in nonlinear dynamical theory become more versatile and favorable [20] [21]. Brain dynamics can be conceived as a large ensemble of coupled nonlinear dynamical subsystems [22] [23] [24] [25]. We reported the nonlinear, chaotic characteristics of Zen-meditation EEG and disclosed significant nonlinear synchronization between EEG channels on a macroscopic scale [26]. This type of nonlinear coupling quantifying the nonlinear interdependence between different cortical areas provides a powerful mechanism for evaluating integrative neural processing [26]-[34]. This study presents a new scheme for probing into the microstate of nonlinear interconnectivity behaviors among various EEG channels and exploring the differences between Zen-meditation and normal resting EEG under the low-alpha oscillatory modes.

\section{Methods}

This paper was aimed at the concept and scheme of constructing the nonlinear interregional-interconnectivity microstate, of which the statistical property may be used to derive the corresponding macrostate. EEG data were collected in 2003 from 8 Zen-meditation practitioners (40 - 55 years old, Zen-meditation experience of 5 - 12 years) and 8 normal, healthy controls (20 - 30 years old). All subjects signed the Informed Consent Form before enrolling in the study and authorized the usage of their data for the scientific analysis. All procedures were consistent with the Declaration of Helsinki and were approved by the Institutional Review Board of National Chiao Tung University.

\subsection{EEG Data}

In the 45-minute recording, Zen practitioners practiced HHIS Zen meditation with all perceptions abiding inside the heart, while the control volunteers were awake but relaxed with eyes closed. EEG signals were recorded by PowerLab/16SP recording system (AD Instruments, Australia), using the 30-channel, common-reference (linked-mastoid MS1-MS2) electrode montage based on the international 10 - 20 system [26]. No particular intervention was applied. In each group, 15 segments of $1 \mathrm{~min}$ low-alpha $(\sim 9 \mathrm{~Hz})$ dominated EEGs were randomly, automatically selected by computer.

\subsection{Signal Pre-Processing}

The EEG signals were sampled at $200 \mathrm{~Hz}$ after being filtered by the analog band-pass filter $(0.5-50 \mathrm{~Hz})$. The segments contaminated by such artifacts as 
eye blinking, eyeball movement and muscle activities were pre-screened. Please do not revise any of the current designations.

\subsection{Nonlinear Interdependence Measure}

The scheme for evaluating the nonlinear interdependence was based on the modified algorithm employed in computing the similarity index $S(\mathbf{X} \mid \mathbf{Y})$ [26]. Major tasks in the algorithm involve reconstruction of the $m$-dimensional phase-space trajectory and computation of the average cloud radius centered at a given state point.

In the phase-space reconstruction, the brain is considered as the nonlinear dynamical system. State variables $\mathbf{X}$ and $\mathbf{Y}$ in the $m$-dimensional phase space are constructed respectively from two channels of EEG signals $x[i]$ and $y[i]$ by Takens embedding theory [23], $\mathbf{X}=\left\{X_{i} \mid X_{i}=(x[i], x[i+\tau], \cdots, x[i+(m-1) \tau]]\right\}_{i=1}^{N}$. The reconstruction assumes a total number of $N$ system-state points in the $m$-dimensional phase-space trajectory, utilizing a rational time delay $\tau$ (in sample). The dimension $m$ indicates the number of degrees of freedom of the nonlinear system and, accordingly, reflects the complexity of the system dynamics.

To compute the average cloud radius, consider a state point $X_{i}$ on the $m$-dimensional phase trajectory. As illustrated in Figure 1, a $K \mathrm{NN}$ hypersphere, formed by the $K$ 's nearest neighbors ( $K \mathrm{NNs})$ of $X_{p}$ is a cloud composed of $K$ neighboring points around $X_{i}$.

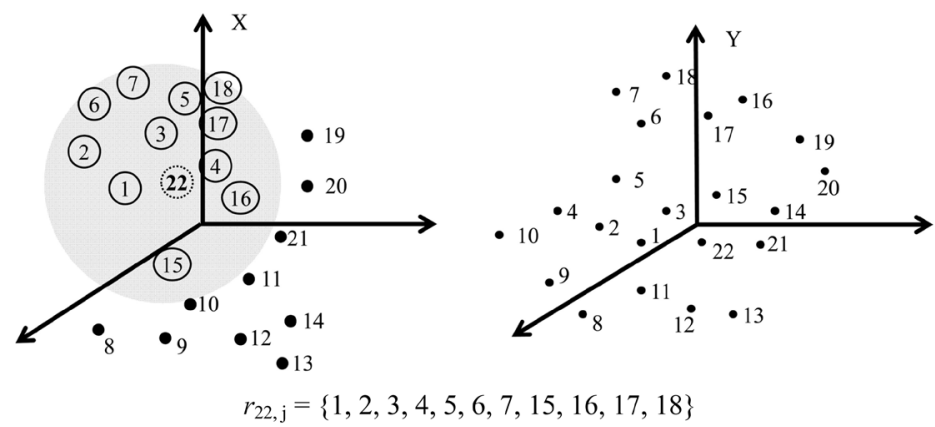

(a)

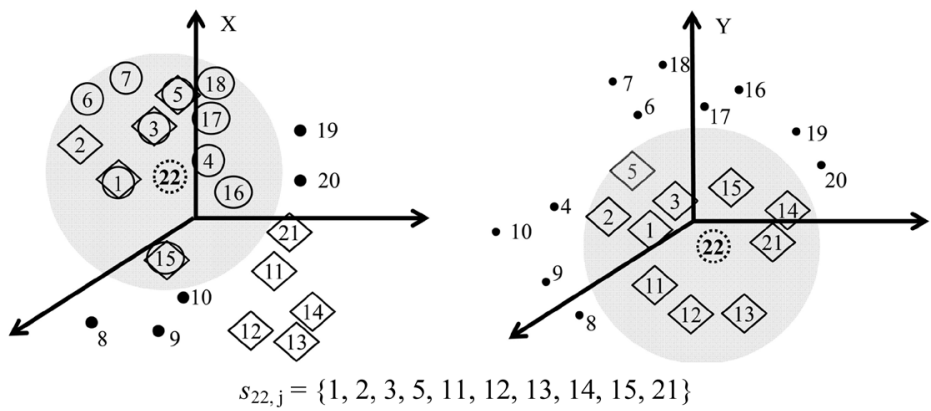

(b)

Figure 1. Ilustration for (a) left: Self neighbors $X_{r_{22, j}}(\bigcirc)$, and right: State points in $\mathrm{Y}$, (b) left: Mutual neighbors $X_{s_{22, j}}(\diamond)$ where the indexes $s_{22, j}$ are determined from the indexes of $K \mathrm{NN}$ of $Y_{22}(K=10)$, assuming $m=3, K=10, i=22$, and $N=22$. 
Let $r_{i, j}$ and $s_{i, p} j=1, \cdots, K$, denote the time indices of the $K \mathrm{NN}$ points of $X_{i}$ and $Y_{i}$, respectively. Note that $r_{i, j}$ and $s_{i, j}$ must be no greater than $i$ for a causal processor. Then, the set of state points in the $K \mathrm{NN}$ hypersphere centered at $X_{i}$ is $\left\{X_{r_{i, j}} \mid j=1, \cdots, K\right\}$. The average square Euclidean distance from $X_{i}$ to its $K \mathrm{NN}$ neighbors (or the average square radius of the cloud centered at $X_{i}$ ) is defined as:

$$
R_{i}(\mathrm{X})=\frac{1}{K} \sum_{j=1}^{K}\left\|X_{i}-X_{r_{i, j}}\right\|^{2}
$$

where $\|\cdot\|$ indicates the operator for calculating the Euclidean distance. Another point cloud around $X_{i}$ is formed with respect to its mutual neighbors $X_{s_{i, j}}$, which share the same temporal indexes of the $K \mathrm{NN}$ of $Y_{i}$. In this sense, the $Y$-conditioned average square Euclidean distance is defined by replacing the true nearest neighbors of $X_{i}$ by the mutual neighbors [26]:

$$
R_{i}(\mathrm{X} \mid \mathrm{Y})=\frac{1}{K} \sum_{j=1}^{K}\left\|X_{i}-X_{s_{i, j}}\right\|^{2}
$$

In the extreme case of $K=N$, the average square radius of the cloud centered at $X_{i}$ becomes

$$
R_{i}^{N}(\mathrm{X})=\frac{1}{N-1} \sum_{j=1, j \neq i}^{N}\left\|X_{i}-X_{j}\right\|^{2}
$$

Then, for two strongly synchronized systems, both self and mutual neighbors mostly coincide so that $R_{i}(\mathrm{X}) \approx R_{i}(\mathrm{X} \mid \mathrm{Y}) \ll R_{i}^{N}(\mathrm{X})$; whereas for independent systems, mutual neighbors are more scattered that leads to $R_{i}(\mathrm{X}) \ll R_{i}(\mathrm{X} \mid \mathrm{Y}) \approx R_{i}^{N}(\mathrm{X})$. Accordingly, the degree of interdependence of these two systems is quantified by the similarity index $S$ [26] between these two cloud patterns formed by self and mutual neighbors, as defined below:

$$
S(\mathrm{X} \mid \mathrm{Y})=\frac{1}{N} \sum_{i=1}^{N} \frac{R_{i}(\mathrm{X})}{R_{i}(\mathrm{X} \mid \mathrm{Y})}
$$

The average over the entire data points in the m-dimensional phase space trajectory can be referred as the macrostate similarity index (maSI). Accordingly, the microstate similarity index (miSI) at a specific time instant $i$ can be defined as the ratio of the average square cloud radius to the average square Y-conditioned cloud radius. That is

$$
S_{i}(\mathrm{X} \mid \mathrm{Y})=\frac{R_{i}(\mathrm{X})}{R_{i}(\mathrm{X} \mid \mathrm{Y})}
$$

$S(\mathrm{X} \mid \mathrm{Y})$ assesses the statistical dependence of the state-space structure of $\mathrm{X}$ on that of $\mathbf{Y}$ in the sense of testifying whether closeness in $\mathbf{X}$ implies closeness in Yand vice versa. Two identical systems with the same sets of self and mutual neighbors result in the maximum similarity index $(S=1)$; whereas the index is close to zero $(S \approx 0)$ for completely independent systems. The opposite interdependence $(S(\mathrm{Y} \mid \mathrm{X}))$ can be computed analogically. Notice that similarity indexes are in general asymmetric, that is, $S(\mathrm{Y} \mid \mathrm{X}) \neq S(\mathrm{X} \mid \mathrm{Y}) . S(\mathrm{X} \mid \mathrm{Y})$ evaluates the effect of system $\mathrm{Y}$ on system $\mathrm{X}$. From the point of view of the system theory, signal $\mathrm{Y}$ is 
regarded as the source or the active role in the interaction; while signal $\mathbf{X}$ plays a passive role (a sink). On the other hand, $S(\mathrm{Y} \mid \mathrm{X})$ analysis considers $\mathrm{Y}$ as the sink that plays the passive role [22].

The asymmetry of $S$ is one of the main advantages over the other nonlinear measures such as the mutual information and the phase synchronizations. The fact that $S$ is asymmetric allows us to study not only topographic patterns but also functional properties. By considering each EEG electrode either as a sink or as a source in the nonlinear-interdependence interaction, we may thereby further explore the brain functional topological profile and the direction of interaction among local neuronal networks [17]. For example, the condition of $S(\mathrm{Y} \mid \mathrm{X})>S(\mathrm{X} \mid \mathrm{Y})$ indicates that $\mathrm{Y}$ depends more on $\mathrm{X}$ than vice versa. In other words, $\mathrm{X}$ is said to be more active and $\mathrm{Y}$ is more passive. By considering each electrode either as a sink or as a source in the nonlinear dynamical interaction, we may explore the spatial direction of the interaction and the dominance of local neuronal networks under Zen meditation [35].

To determine the implementation parameters, we employed mutual information function in determining the appropriate time delay. The suitable range of tis 5 - 7 samples. Embedding dimension $m$ can be determined by the convergent estimate of dimension. To ensure the completeness of system dynamics, the suitable range of $m$ is 8 - 10 using Cao's method to explore the system dynamics under various $m$ 's. There is no analytical approach for determining the value of $K$ in constructing the $K \mathrm{NN}$ hypersphere. When $K$ is small $(K \leq 4)$, the neighborhoods in one reconstructed trajectory can map into the other perfectly. The strength of nonlinear interdependence between two EEG trajectories is so strong that $S$ values are close to 1 . Likewise, if $K$ is large enough to cover the most points in phase space, it causes strong strength of nonlinear interdependence since the $X$-neighbor and $X / Y$-mutual neighbor cloud radii overlap with each other. Values of $K$ ranging from 5 to 20 lead to a consistent estimate of similarity index. To reduce the computational load, $K=6$ is adopted. The window length $N$ is selected to encompass the stabilization of dynamical behavior in the sense of the convergent estimate of EEG nonlinear dynamical property. The window of 2 - 5 seconds was applied previously when studying the macroscopic behavior. To explore the microstate nonlinear dynamics of brain functioning, the window size of 1 second (200 samples) is adopted. Note that the 1-second window is the $K \mathrm{NN}$ searching range in (1) and (2), indicating the influence of past events on current system state. The miSI in (5) is computed every 0.005 second for a given cluster center. Finally, the implementing parameters were: $\tau=6$ (sample points), $m=8, K=6$ and $N=200$ [26] [36].

\subsection{Microstate SIM Clustering}

Interpretation of long-term, multichannel EEG record has been one of the most important tasks in investigating the time-varying brain dynamics under the specific physical, mental, conscious, or cognitive states. Before interpreting the EEG record, the first step is normally to identify the possible brain dynamical charac- 
teristics modeled by specific representations, mostly in quantitative form. Different representations are designed to emphasize different brain behaviors including spatial-temporal focalization of EEG rhythms, topographical mapping of cortical potentials, efficiency of inter-regional neural-network interactions, etc. Such problem is referred to the area of knowledge discovery in databases (KDD), or data mining.

Among various clustering methods, $K$-means clustering based on minimizing a specified objective function might be the most widely used method [37]. $K$-means clustering classifies the $n$ elements into $k$ sets, $\mathbf{S}=\left\{S_{1}, S_{2}, \cdots, S_{k}\right\}$ by way of minimizing the within-cluster sum of squared differences between each element and the cluster's center:

$$
\delta=\sum_{i=1}^{k} \sum_{x_{j} \in S_{i}}\left(x_{j}-m_{i}\right)^{2}
$$

where center $m_{i}$ is the average of the elements in the ith cluster $S_{i}$. Iterative refinement technique is implemented. Given an initial set of kcenters $m_{1}, m_{2}, \ldots$, and $m_{k}$. The algorithm proceeds by alternating between two steps, classification and updating step. In classification step, each element $\left(x_{p}\right)$ is assigned to the cluster of which the center is closest to $x_{p}$.

$$
S_{i}^{(t)}=\left\{x_{p} \mid\left\|x_{p}-m_{i}^{(t)}\right\|^{2} \leq\left\|x_{p}-m_{j}^{(t)}\right\|^{2}, \forall j, 1 \leq j \leq k\right\}
$$

where $t$ is the number of iteration. In updating step, the new cluster centers $\left\{m_{p}\right.$ $1 \leq i \leq k\}$ are calculated as below.

$$
m_{i}^{(t+1)}=\frac{1}{N_{i, t}} \sum_{x_{j} \in S_{i}^{(t)}} x_{j}
$$

where $N_{i, t}$ is the number of elements in the cluster $i$ at the $t^{\text {th }}$ iteration. The algorithm converges when the classification settles, that is, no more alteration of cluster elements.

Microstate similarity index (miSI) computed by (5) is evaluated for all $30 \times 30$ EEG channel pairs from which we form the miSIM (microstate similarity index matrix). All the miSIMs in a given time interval (one minute, in this study) are classified by $K$-means clustering algorithm. Totally 12,000 miSIM features in one-minute EEG are classified into five clusters by $K$-means clustering. The final step is to screen out approximately $30 \%$ of the feature vectors which are too far away from each cluster center. The final cluster centers are computer by averaging all the miSIMs inside the cluster. The average miSIM is simply the sum of all miSIMs divided by the total number of miSIMs in the specified pool.

\subsection{Macrostate SIM}

The center of each cluster is the macrostate SIM (maSIM) of the miSIM cluster. We discovered the maSIM of the largest cluster could represent the SIM derived from the conventional method [26] in (4). The advantage of analyzing microstate SIM is to access the flashlight transient behavior of brain dynamics down to 
the millisecond scale. The cluster centers may be used as the feature bases (templates) to interpret the nonlinear interdependence microstates of long-term EEG record. Each miSIM is classified into the cluster of which its center is nearest to the miSIM. Figure 2 displays the template of maSIM, with each row (column) indicating the efficacy of the passive (active) role of the channel on the vertical (horizontal) coordinate.

\subsection{Connectivity Plot}

We designed the connectivity plot to demonstrate the dominant interconnectivity strength among inter-regional neural networks. The connectivity plot also reflects the lateral and longitudinal symmetrical property. The major scheme is to connect two EEG electrode sites with a line if the source-role miSIM $\geq$ threshold (0.6). To make the plot more informative and versatile, different line forms and colors may be used to indicate the spatial relation. In the longitudinal connectivity plot (LCP), EEG channels are divided into frontal (Fp1, Fp2, F7, F3, Fz, F4, F8, FT7, FC3, FCz, FC4, FT8), central (T7, C3, Cz, C4, T8), and posterior (TP7, $\mathrm{CP} 3, \mathrm{CPz}, \mathrm{CP} 4, \mathrm{TP} 8, \mathrm{P} 7, \mathrm{P} 3, \mathrm{Pz}, \mathrm{P} 4, \mathrm{P} 8, \mathrm{O} 1, \mathrm{Oz}, \mathrm{O} 2)$ groups. In the transversal connectivity plot (TCP), EEG channels are divided into left (Fp1, F7, F3, FT7, FC3, T7, C3, TP7, CP3, P7, P3, O1), right (Fp2, F4, F8, FC4, FC8, C4, T8, CP4, TP8, P4, P8, O2), and midline ( $\mathrm{Fz}, \mathrm{FCz}, \mathrm{Cz}, \mathrm{CPz}, \mathrm{Pz}, \mathrm{Oz})$ groups. LCP emphasizes the longitudinal relationship, whereas TCP reflects the dominant connectivity between two hemispheres. The LCP and TCP provide the profile of high

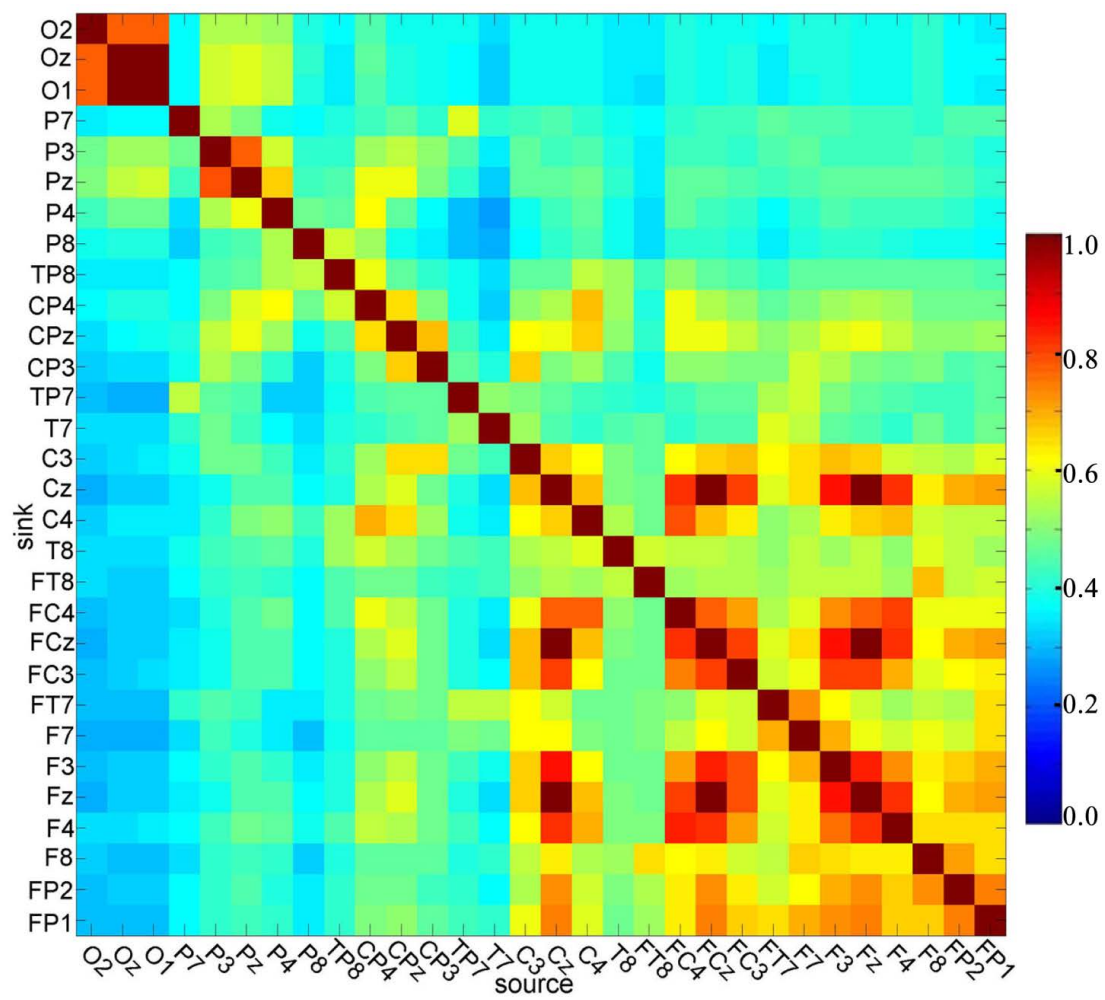

Figure 2. MaSIM template (the second cluster center for Zen-meditation EEG). 
interdependence ( $S I \geq 0.6)$ for channel pairs with the frontal-posterior and left-right relation. Red (blue) lines in LCP indicate the source locates in the frontal (posterior) region, while green lines mark the strong interdependence of channel pairs with the source on the transversal line (T3-C3-Cz-C4-T4). In the TCP plot, red (blue) lines indicate the source locates in the right (left) hemisphere, while green lines indicate the source locates in the midline.

\section{Results}

The miSIMs for one-minute Zen-meditation and resting EEG are classified into five clusters. Figure 3 and Figure 4 displays the cluster centers (last row) and 3 sampled cluster members (miSIMs, on the top three rows) in each of the five clusters, respectively, for Zen-meditation EEG and resting EEG. The probabilities of occurrence of each individual cluster are $13.6 \%$ (16.4\%), 11.5\% (10.2\%), $23.8 \%(28.0 \%), 30.4 \%(32.5 \%)$ and $20.7 \%(12.9 \%)$ in a 5 -minute Zen-meditation (resting) EEG. The second cluster appears least often in both EEG cases, nevertheless, contains the most active miSIMs. The most inactive miSIMs are in the first (fifth) cluster for Zen-meditation (resting) EEG.

In Figure 3 and Figure 4, all clusters contains the frontal-central focalized (channels $\mathrm{Cz}, \mathrm{FCz}, \mathrm{Fz}$ ) miSIMs. The miSIMs in the first and fifth clusters exhibit comparably suppressed inter-channel activities. The second cluster contains the most active regional interconnectivity of miSIMs, with the activity in the centro-parietal regions increasing moderately. In the third cluster, spatial characteristic of higher interdependence is very similar to which of the first cluster. Nevertheless, miSIMs in the third cluster have larger regions of mid-level $(0.3 \leq$

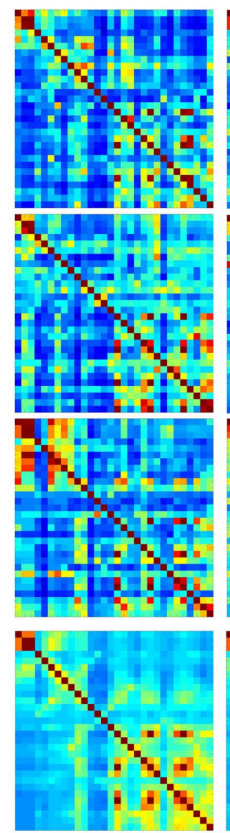

$1^{\text {st }}$ cluster
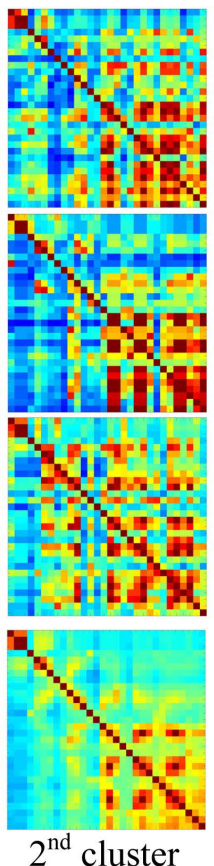
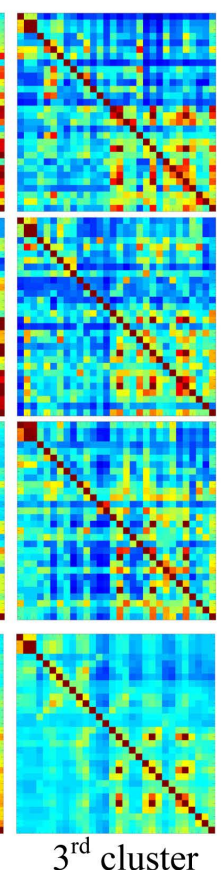

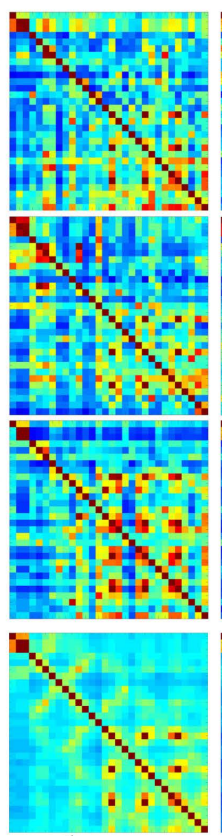

$4^{\text {th }}$ cluster

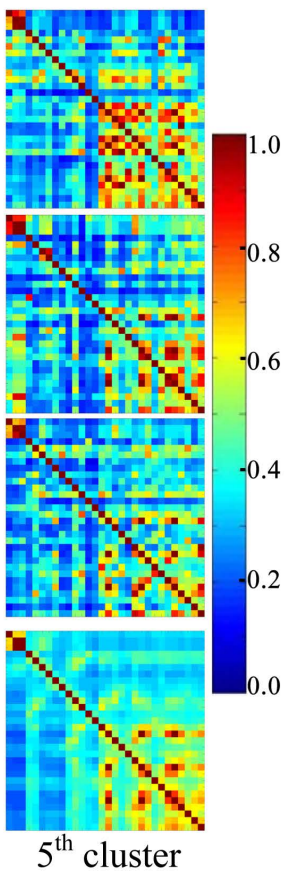

Figure 3. Rows 1 - 3 display 3 sampled miSIMs in each of the five clusters for Zen-meditation EEG, the last row display the corresponding cluster centers (maSIMs). 


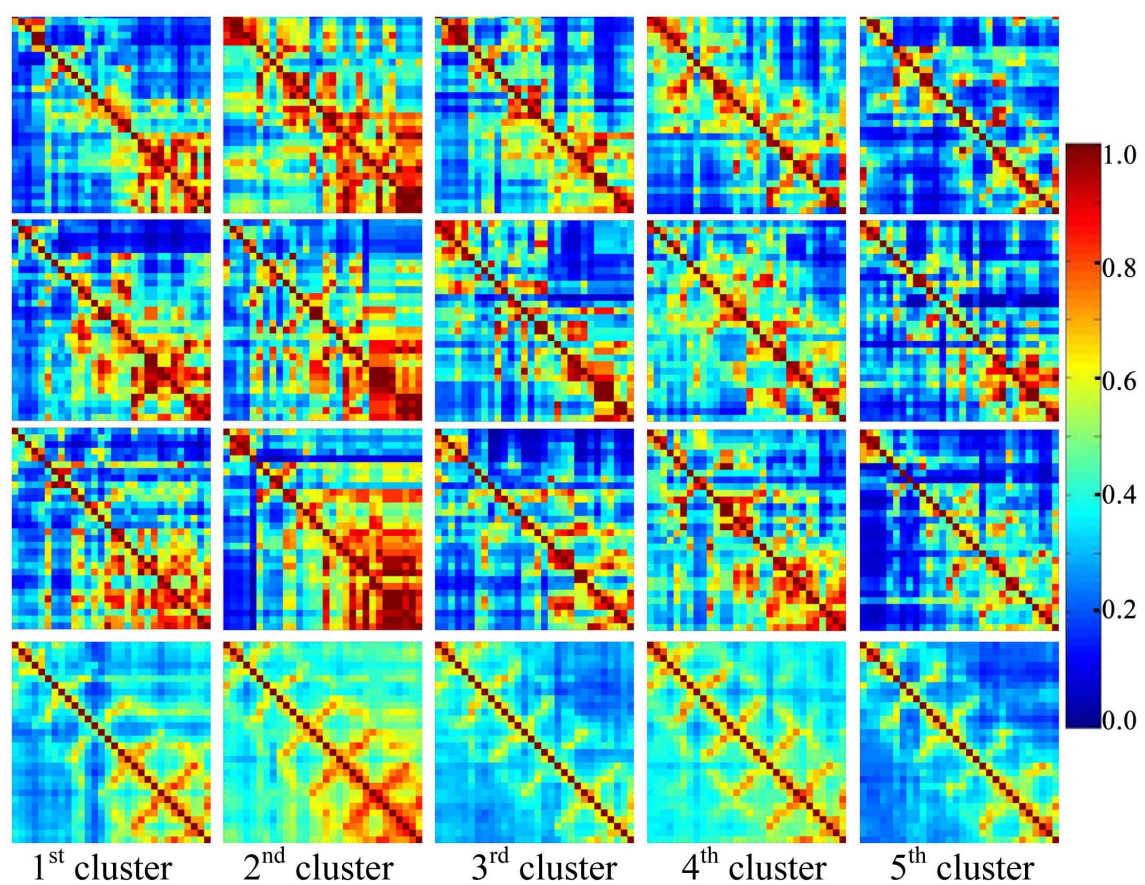

Figure 4. Rows 1 - 3 display 3 sampled miSIMs in each of the five clusters for resting EEG, the last row display the corresponding cluster centers (maSIMs).

SI $\leq 0.5$, light blue in Figure 3) strengths of interdependence. The dynamics of nonlinear interdependence of the fourth cluster is more vigorous than which of the first and the third cluster, yet, is inactive compared with the second cluster possessing highest extending capability in the frontal channels. The overall nonlinear interdependence of the fifth cluster is similar to which of the fourth cluster in the frontocentral regions, yet, becomes more inactive in the temporo-parietal and occipital regions.

Zen-meditation EEG exhibits right-dominant, while resting EEG does not. The active regions of resting EEG (with SI $>0.6$, in the connectivity plots) are more dispersive that extend to inferior frontal and temporal cortexes. Comparison of Figure 3 and Figure 4 reveals that Zen-meditation brain exhibits rather constraint, localized characteristics of regional interconnectivity, while normal brain at rest tends to extend the strength of influence to other regional neural networks. Moreover, resting EEG demonstrates the remarkable expansion of source-driven effect towards the inferior brain regions (EEG channels F7, F8, FT7, FT8, T7, T8, TP7 and TP8). The phenomenon may be clearly illustrated by connectivity plots (LCP and TCP) in Figure 5.

Table 1 lists the averages of source-role (active) miSIMs in 5 brain regions for each cluster (Zen-meditation/resting EEG). Regional average smears out the statistical difference between two groups ( $p<0.05$ only for cluster 5$)$. The largest microstate SI average appears in the right frontal (0.549) and central (0.568) region (the $2^{\text {nd }}$ cluster) for Zen-meditation EEG; while the smallest value appears in the left posterior region $(0.327$ in the first cluster and 0.349 in the fifth cluster). Notice that, among all five clusters, right hemisphere always exhibits higher 


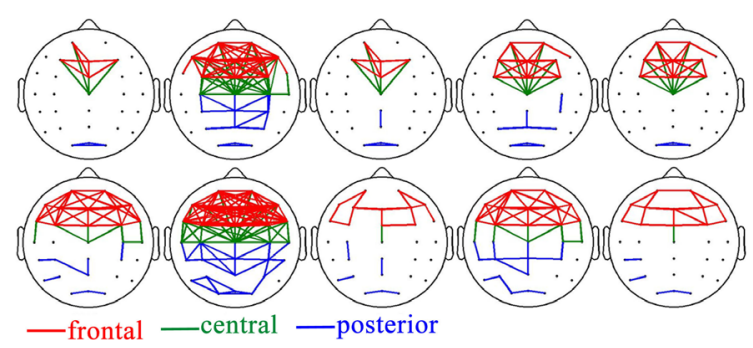

(a)

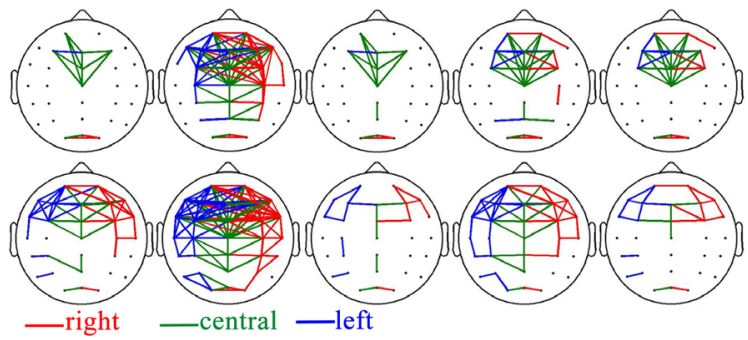

(b)

Figure 5. (a) LCP and (b) TCP for Zen-meditation EEG (top) and resting EEG (bottom).

Table 1. Averages of source-role (active) miSIMs in 5 brain regions for each cluster (Zen-meditation/resting EEG).

\begin{tabular}{cccccc}
\hline $\begin{array}{c}\text { Cluster } \\
\text { Brain region }\end{array}$ & 1 & 2 & 3 & 4 & 5 \\
\hline Left Frontal & $0.37 / 0.46$ & $0.53 / 0.57$ & $0.39 / 0.40$ & $0.45 / 0.47$ & $0.44 / 0.37$ \\
Right Frontal & $0.39 / 0.47$ & $0.55 / 0.57$ & $0.40 / 0.39$ & $0.46 / 0.47$ & $0.45 / 0.37$ \\
Central & $0.42 / 0.43$ & $0.57 / 0.55$ & $0.45 / 0.40$ & $0.50 / 0.47$ & $0.47 / 0.36$ \\
Left Posterior & $0.33 / 0.33$ & $0.44 / 0.46$ & $0.37 / 0.38$ & $0.41 / 0.42$ & $0.35 / 0.32$ \\
Right Posterior & $0.35 / 0.37$ & $0.46 / 0.46$ & $0.39 / 0.37$ & $0.42 / 0.42$ & $0.36 / 0.31$ \\
\hline
\end{tabular}

interdependence strength than left hemisphere, regardless of the frontal or posterior region. Such a right-dominant behavior is apparent in the second cluster (Figure 5(b)). In respect of the longitudinal relation, the frontal region apparently dominates over the posterior region in both left and right hemispheres for all the five clusters.

In resting EEG, the largest miSIM average appears in the right frontal (0.570) and left frontal (0.574) region (the $2^{\text {nd }}$ cluster); while the smallest value appears in the posterior region $(0.317$ in the left posterior and 0.312 in the right posterior, both belonging to the fifth cluster). With respect to the lateral symmetry, there exists no consistent laterally dominant behavior among all five clusters, unlike the right dominant characteristics in all five cluster centers for the Zen-meditation practitioner. With respect to the longitudinal relation, the frontal region apparently dominates over the posterior region in both left and right hemispheres for all the five clusters.

Zen-meditation miSIM appears to be more localized with the major activity in the midline ( $\mathrm{Fz}, \mathrm{FCz}$, and $\mathrm{Cz}$ ). On the other hand, rest miSIM is more outspread with the major activity extending to inferior brain area. We compare the miSIMs 
in the $4^{\text {th }}$ cluster between Zen-meditation and resting EEG since the $4^{\text {th }}$ cluster is the largest cluster. Figure 6 illustrates the brain mappings of miSIMs, in the $4^{\text {th }}$ cluster, of Zen-meditation (left) and resting (right) EEG given the source locating at channel: (a) C3, (b) Cz, (c) F8, (d) P3, and (e) T8.

\section{Discussion}

Figure 6 demonstrates noticeable distinction between Zen-meditation and rest EEG ( $p$ value $<<0.05$ ) although they both are mainly composed of low alpha ( 8 $10 \mathrm{~Hz}$ ) activities. Channels $\mathrm{C} 3$ and $\mathrm{C} 4$ are in charge of sensorimotor integration for the right and left body. While entering into deep Zen meditation, practitioners transcend the time-space perception and cognition. Channels P3 and P4 associated with spatiotemporal information processing of right half and left half of space appear to be inactive for Zen-meditation practitioners. Brain function near EEG channel F8 correlates with emotional expression. In addition, remarkable distinction of spatially nonlinear interconnectivity was observed at temporal lobe (Figure 6(e)) correlating with auditory processing. Zen-meditation practitioners having activated ChanXin mailun (inside the third ventricle) are able to focalize the brain activities within the smaller regions around the midline. Channels $\mathrm{Fz}, \mathrm{FCz}$ and $\mathrm{Cz}$ interact tightly (Figure 6(b)). Accordingly, practitioners can easily transcend the interference from sensory organs and emotions. Other channels beyond the midline region are much quieter for Zen-meditation practitioners, yet, are rather vigorous for the normal people at rest.

\section{Conclusions}

This paper reports a novel scheme of investigating the microstates of spatially nonlinear interdependence among EEG channels. Previous researches have been focused on the macrostate analysis of EEG nonlinear interdependence [22] [32]. Averaging the raw SI's (similarity indexes) over an epoch of (for example, 2 seconds) results in the obscurity of possibly important regional neural-network interactions. Microstate analysis down to millisecond scale allows us to explore

(a)

(b)
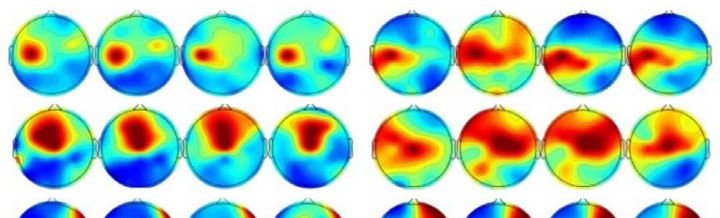

(c)

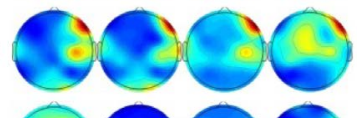

(d)
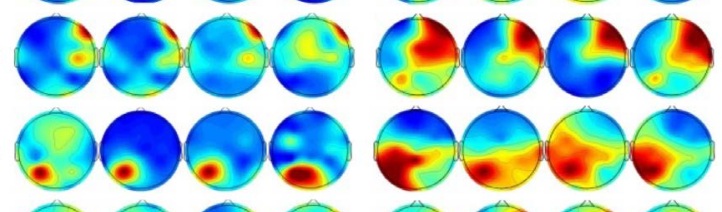

(e)

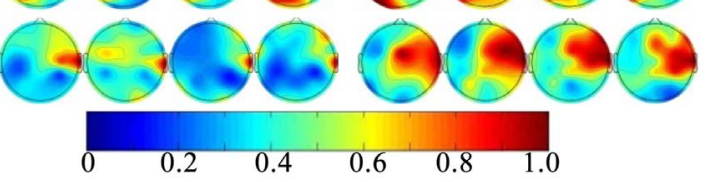

Figure 6. Brain mappings of active miSIMs for (left) Zen-meditation EEG, and (right) resting EEG, assume the source channel is (a) C3; (b) Cz; (c) F8; (d) P3 and (e) T8. 
microscopic transient behaviors. As illustrated in Figure 7, brain mappings of maSIMs, in the $4^{\text {th }}$ cluser, reveal slight difference between Zen-meditation EEG (left) and resting EEG (right). Nevertheless, macrostate SIM is deficient in expressing the transient dynamics of time-varying interregional connectivity in comparison with microstate SIMs in Figure 6. Student T-test shows statistically significant difference in maSIMs (Figure 7) between Zen-meditation and resting EEG ( $p$ value $<0.05)$.

The analysis found significant differences of interregional-connectivity microstates between Zen-meditation EEG and resting EEG, particularly in the regions known to be involved in sensorimotor function, cognitive and spatial perceptions, emotion and mood regulation, and verbal and auditory processing. In preparation for entering true Zen realm, brain needs to switch to the detached state so that the spiritual heart starts to take over. Zen practitioners accomplish such a conversion by simultaneously abiding in the third ventricle of the brain, the heart, and the perineum [18] [38] which leads to the so-called Heaven-Man-Earth harmonious resonance in Zen. The concrete, tight connection among $\mathrm{Fz}, \mathrm{FCz}$ and $\mathrm{Cz}$ (Figure 6(b) left) provides the evidence of the effect of tri-mailuns unification. Brain in such a state is totally tranquil and peaceful, being relieved from the routine processing of the external messages from sensory organs and void of all the wandering thoughts, cognitions, or mental activities. Somehow this finding is resonating with papers on EEG effects of meditation, though using different mediation techniques [39].

In comparison of the results, Zen-meditation miSIM appears to be more focalized in the midline ( $\mathrm{Fz}, \mathrm{FCz}$, and $\mathrm{Cz}$ ), whereas resting miSIM is more outspread with the major activity extending to inferior brain regions. Zen-meditation miSIM has higher interdependence only in a smaller area mainly in the fronto-central region (Cz-F3: 0.76, Cz-F4: 0.71, FCz-F3: 0.77, FCz-F4: 0.71). Resting miSIM has higher regional interdependence in the electrode pairs of nearby EEG channels, particularly in the fronto-temporal regions (FT7-T7: 0.67, FT8-T8: 0.61, FC3-C3: 0.62, FC4-C4: 0.63). In addition, resting miSIM has more activities in the occipital region. Comparing the miSIM cluster with the lowest activity $\left(1^{\text {st }}\right.$ for Zen-meditation EEG and $5^{\text {th }}$ for resting EEG), Zen-meditation miSIM has very low activity outside the fronto-central region $(\mathrm{Cz}, \mathrm{FCz}$ and $\mathrm{Fz})$, while resting miSIM still involves significant activities in such regions like F7, F8, F3 and $\mathrm{F} 4$, as illustrated in Table 2 . The $\mathrm{T}$ test shows statistically significant difference between two groups with $p<0.05$.

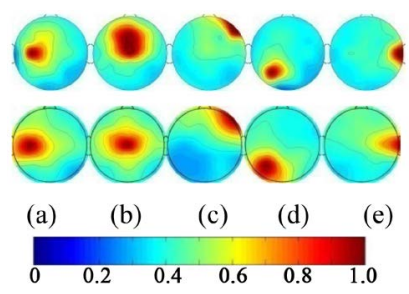

Figure 7. Brain mappings of maSIMs of Zen-meditation EEG (up) and resting EEG (down) assume the source channel is (a) C3; (b) Cz; (c) F8; (d) P3; and (e) T8. 
Table 2. Averages of microstate similarity indexes of the specified channel pairs in the $1^{\text {st }}$ cluster for Zen-meditation EEG and which in the $5^{\text {th }}$ cluster for resting EEG.

\begin{tabular}{cccccc}
\hline $\begin{array}{c}\text { Channel } \\
\text { pairs }\end{array}$ & $\begin{array}{c}\text { Zen } \\
\text { meditation }\end{array}$ & Resting & $\begin{array}{c}\text { Channel } \\
\text { pairs }\end{array}$ & $\begin{array}{c}\text { Zen } \\
\text { meditation }\end{array}$ & Resting \\
\hline F7-FT7 & 0.49 & 0.79 & F8-FT8 & 0.53 & 0.75 \\
F7-F3 & 0.47 & 0.66 & F8-F4 & 0.49 & 0.74 \\
F7-Fp1 & 0.44 & 0.74 & F8-Fp2 & 0.56 & 0.8 \\
F3-FC3 & 0.57 & 0.77 & F4-FC4 & 0.54 & 0.75 \\
F3-F7 & 0.51 & 0.67 & F4-F8 & 0.51 & 0.75 \\
F3-Fp1 & 0.59 & 0.78 & F4-Fp2 & 0.50 & 0.78 \\
\hline
\end{tabular}

In the results of analyzing Zen-meditation EEG, profoundly experienced practitioners, after the ignition of ChanXin inside the third ventricle, reveal macroscopic behaviors of brain dynamics with a remarkable stationary oscillation mode among all regional neural oscillators that have been tuned in with the particular resonance via heart-to-heart imprint practice in Zen meditation. On the other hand, microscopic activities involve subtle transitions of effectiveness among regional neural oscillators, in our hypothesis, to modulate the brain neural dynamics for reducing the mental activities, cleansing the subliminal consciousness, and transcending the time- and space-dependent physical world.

\section{Acknowledgements}

This research was supported by the grants from the Ministry of Science and Technology of Taiwan (Grant No.: MOST 104-2221-E-009-189-MY2).

\section{References}

[1] Lehmann, D., Faber, P.L., Galderisi, S., Herrmann, W.M., Kinoshita, T., Koukkou, M., et al. (2005) EEG Microstate Duration and Syntax in Acute, Medication-Naïve, First-Episode Schizophrenia: A Multi-Center Study. Psychiatry Research: Neuroimaging, 138, 141-156. https://doi.org/10.1016/j.pscychresns.2004.05.007

[2] Koenig, T., Kochi, K. and Lehmann, D. (1998) Event-Related Electric Microstates of the Brain Differ between Words with Visual and Abstract Meaning. Electroencephalography and Clinical Neurophysiology, 106, 535-546. https://doi.org/10.1016/S0013-4694(97)00164-8

[3] Fingelkurts, A.A., Fingelkurts, A.A., Bagnato, S., Boccagni, C. and Galardi, G. (2012) EEG Oscillatory States as Neuro-Phenomenology of Consciousness as Revealed from Patients in Vegetative and Minimally Conscious States. Consciousness and Cognition, 21, 149-169. https://doi.org/10.1016/j.concog.2011.10.004

[4] Fingelkurts, A.A., Fingelkurts, A.A. and Neves, C.F. (2010) Natural World Physical, Brain Operational, and Mind Phenomenal Space-Time. Physics of Life Reviews, 7, 195-249. https://doi.org/10.1016/j.plrev.2010.04.001

[5] Freeman, W.J. (2004) Origin, Structure, and Role of Background EEG Activity, Part 1. Analytic Amplitude. Clinical Neurophysiology, 115, 2077-2088. https://doi.org/10.1016/j.clinph.2004.02.029 
[6] Yu, T., Tsai, H.L. and Hwang, M.L. (2003) Suppressing Tumor Progression of In Vitro Prostate Cancer Cells by Emitted Psychosomatic Power through Zen Meditation. The American Journal of Chinese Medicine, 31, 499-507. https://doi.org/10.1142/S0192415X03001132

[7] Lester, D. (1999) Zen and Happiness. Psychological Reports, 84, 650-651. https://doi.org/10.2466/pr0.1999.84.2.650

[8] MacLean, C.R., Walton, K.G., Wenneberg, S.R., Levitsky, D.K., Mandarino, J.P., Waziri, R., et al. (1997) Effects of the Transcendental Meditation Program on Adaptive Mechanisms: Changes in Hormone Levels and Responses to Stress after 4 Months of Practice. Psychoneuroendocrinology, 22, 277-295. https://doi.org/10.1016/S0306-4530(97)00003-6

[9] Tooley, G.A., Armstrong, S.M. and Norman, T.R. (2000) Acute Increases in Night-Time Plasma Melatonin Levels Following a Period of Meditation. Biological Psychology, 53, 69-78. https://doi.org/10.1016/S0301-0511(00)00035-1

[10] Fingelkurts, A.A., Fingelkurts A.A. and Kallio-Tamminen, T. (2015) EEG-Guided Meditation: A Personalized Approach. Journal of Physiology-Paris, 109, 180-190. https://doi.org/10.1016/j.jphysparis.2015.03.001

[11] Tang, Y.Y., Ma, Y.H. and Fan, Y.X. (2009) Central and Autonomic Nervous System Interaction Is Altered by Short-Term Meditation. Proceedings of the National Academy of Sciences of the United States of America, 106, 8865-8870. https://doi.org/10.1073/pnas.0904031106

[12] Lutz, A., Slagter, H.A. and Rawlings, N.B. (2009) Mental Training Enhances Attentional Stability: Neural and Behavioral Evidence. Journal of Neuroscience, 29, 13418-13427. https://doi.org/10.1523/JNEUROSCI.1614-09.2009

[13] Bertisch, S.M., Wee, C.C., Phillips, R.S. and McCarthy, E.P. (2009) Alternative Mind-Body Therapies Used by Adults with Medical Conditions. Journal of Psychosomatic Research, 66, 511-519. https://doi.org/10.1016/j.jpsychores.2008.12.003

[14] Marchand, W.R. (2012) Mindfulness-Based Stress Reduction, Mindfulness-Based Cognitive Therapy, and Zen Meditation for Depression, Anxiety, Pain, and Psychological Distress. Journal of Psychiatric Practice, 18, 233-252. https://doi.org/10.1097/01.pra.0000416014.53215.86

[15] Wu Jue, M.T. (2010) Wisdom and the Guide to Heart Chan Meditation. lulu.com.

[16] Fingelkurts, A.A. and Fingelkurts, A.A. (2014) EEG Oscillatory States: Universality, Uniqueness and Specificity across Healthy-Normal, Altered and Pathological Brain Conditions. PLoS ONE, 9, e87507. https://doi.org/10.1371/journal.pone.0087507

[17] Cahn, B.R. and Polich, J. (2006) Meditation States and Traits: EEG, ERP, and Neuroimaging Studies. Psychological Bulletin, 132, 180-211. https://doi.org/10.1037/0033-2909.132.2.180

[18] Travis, F. and Shear, J. (2010) Focused Attention, Open Monitoring and Automatic Self-Transcending: Categories to Organize Meditations from Vedic, Buddhist and Chinese Traditions. Consciousness and Cognition, 19, 1110-1118. https://doi.org/10.1016/j.concog.2010.01.007

[19] Lo, P.C., Huang, M.L. and Chang, K.M. (2003) EEG Alpha Blocking Correlated with Perception of Inner Light during Zen Meditation. The American Journal of Chinese Medicine, 31, 629-642. https://doi.org/10.1142/S0192415X03001272

[20] Arnhold, J., Grassberger, P., Lehnertz, K. and Elger, C. (1999) A Robust Method for Detecting Interdependencies: Application to Intracranially Recorded EEG. Physica D, 134, 419-430. https://doi.org/10.1016/S0167-2789(99)00140-2 
[21] St. Louis, E.K. and Lansky, E.P. (2006) Meditation and Epilepsy: A Still Hung Jury. Med Hypotheses, 67, 247-250. https://doi.org/10.1016/j.mehy.2006.02.039

[22] Aftanas, L.I. and Golocheikine, S.A. (2001) Human Anterior and Frontal Midline Theta and Lower Alpha Reflect Emotionally Positive State and Internalized Attention: High-Resolution EEG Investigation of Meditation. Neuroscience Letters, 310, 57-60. https://doi.org/10.1016/S0304-3940(01)02094-8

[23] Gans, F., Schumann, A.Y., Kantelhardt, J.W., Penzel, T. and Fietze, I. (2009) Cross-Modulated Amplitudes and Frequencies Characterize Interacting Components in Complex Systems. Physical Review Letters, 102, Article ID: 098701. https://doi.org/10.1103/PhysRevLett.102.098701

[24] Bhattacharya, J., Petsche, H. and Pereda, E. (2001) Interdependencies in the Spontaneous EEG While Listening to Music. International Journal of Psychophysiology, 42, 287-301. https://doi.org/10.1016/S0167-8760(01)00153-2

[25] Stam, C.J. (2005) Nonlinear Dynamical Analysis of EEG and MEG: Review of an Emerging Field. ClinNeurophysiol, 116, 2266-2301. https://doi.org/10.1016/j.clinph.2005.06.011

[26] Lo, P.C. and Chang, C.H. (2013) Spatially Nonlinear Interdependence of Alpha-Oscillatory Neural Networks under Chan Meditation. Evidence-Based Complementary and Alternative Medicine, 2013, Article ID: 360371. https://doi.org/10.1155/2013/360371

[27] Takens, F. (1981) Detecting Strange Attractors in Turbulence. In: Rand, D.A. and Young, L.-S., Eds., Dynamical Systems and Turbulence, Lecture Notes in Mathematics, Vol. 898, 366-381. https://doi.org/10.1007/BFb0091924

[28] Calitoiu, D., Oommen, B.J. and Nussbaum, D. (2012) Large-Scale Neuro-Modeling for Understanding and Explaining Some Brain-Related Chaotic Behavior. Simulation: Transactions of the Society for Modeling and Simulation International, 88, 1316-1337. https://doi.org/10.1177/0037549712450331

[29] Fabrice, W., Karim, A.A., Fabrice, B. and Lotfi, S. (2009) From EEG Signals to Brain Connectivity A Model-Based Evaluation of Interdependence Measures. Journal of Neuroscience Methods, 183, 9-18. https://doi.org/10.1016/j.jneumeth.2009.04.021

[30] Breakspear, M. and Terry, J.R. (2002) Detection and Description of Non-Linear Interdependence in Normal Multichannel Human EEG Data. Clinical Neurophysiology, 113, 735-753. https://doi.org/10.1016/S1388-2457(02)00051-2

[31] Breakspear, M. and Terry, J.R. (2002) Topographic Organization of Nonlinear Interdependence in Multichannel Human EEG. Neuroimage, 16, 822-835. https://doi.org/10.1006/nimg.2002.1106

[32] Stam, C.J., Breakspear, M., van Cappellen van Walsum, A.M. and van Dijk, B.W. (2003) Nonlinear Synchronization in EEG and Whole-Head MEG Recordings of Healthy Subjects. Human Brain Mapping, 19, 63-78. https://doi.org/10.1002/hbm.10106

[33] Feldmann, U. and Bhattacharya, J. (2004) Predictability Improvement as an Asymmetrical Measure of Interdependence in Bivariate Time Series. International Journal of Bifurcation and Chaos, 14, 505-514. https://doi.org/10.1142/S0218127404009314

[34] Rubinov, M., Knock, S.A., Stam, C.J., Micheloyannis, S., Harris, A.W.F., Williams, L.M. and Breakspear, M. (2009) Small-World Properties of Nonlinear Brain Activity in Schizophrenia. Human Brain Mapping, 30, 403-416. https://doi.org/10.1002/hbm.20517

[35] Quiroga, R.Q., Kraskov, A., Kreuz, T. and Grassberger, P. (2002) Performance of Different Synchronization Measures in Real Data: A Case Study on Electroence- 
phalographic Signals. Physical Review E, 65, Article ID: 041903. https://doi.org/10.1103/PhysRevE.65.041903

[36] Liu, C.Y. and Lo, P.C. (2008) Spatial Focalization of Zen-Meditation Brain Based on EEG. Journal of Biomedical Engineering Research, 29, 17-24.

[37] Shirwaikar, R. and Bhandari, C. (2013) K-Means Clustering Method for the Analysis of Log Data. 2013 Information Technology and Power Electronics, ACEEE, A Computer Division of IDES.

[38] Lo, P.C. and Huang, M.L. (2015) Scientific Propositions for Brain Reformation by Mailun Chan Ding. International Journal of Electrical and Electronics Engineering, 4, 2278-9952.

[39] Fingelkurts, A.A. and Kallio-Tamminen, T. (2016) Long-Term Meditation Training Induced Changes in the Operational Synchrony of Default Mode Network Modules during a Resting State. Cognitive Processing, 17, 27-37.

https://doi.org/10.1007/s10339-015-0743-4 